\title{
Computer Analysis of Images and Patterns
}

\author{
Pedro Real
}

Published online: 3 July 2013

(c) Springer Science+Business Media New York 2013

This special issue comprises thirteen recent and relevant works in different aspects of the computer analysis of images and patterns. We cover important topics like stereo image processing, image and shape representation, color texture classification, parallel systems and membrane computing, face recognition, pedestrian classification, estimation of photometry and analysis of medical images.

The papers have been divided into four groups - three are based on similarities in the addressed problems and the last focuses on six different applications.

The first section presents stereo image processing from a variety of perspectives.

"Tuning of Adaptive Weight Depth Map Generation Algorithms" by Acosta et al. shows a systematic statistical approach for the parameter setting problem in disparity map estimation within the context of depth map generation algorithms.

The next paper, "P-System Implementation of Dynamic Programming Stereo" by Gimel'farb et al. discusses the capabilities of the main tools of Membrane Computing, the P-systems, and how to implement the symmetric dynamic programming stereo (SDPS) matching algorithm.

The authors propose a novel method of stereo matching based on a combination of local edge detection, cost aggregation computation and belief propagation optimization techniques in "Stereo Matching Based on Dissimilar Intensity Support and Belief Propagation" by Da et al.

The next section studies representation and estimation of shape from different mathematical points of view.

P. Real $(\bowtie)$

Dpto. de Matemática Aplicada I, Escuela Técnica Superior de Ingeniería Informática, Universidad de Sevilla, Seville, Spain e-mail: real@us.es
"Statistical Model of Shape Moments with Active Contour Evolution for Shape Detection and Segmentation" by Zhang et al. describes a novel statistical shape model suitable for robust active contour segmentation.

The following paper, "A Novel Space Variant Image Representation" by Onkarappa et al., proposes to change image sampling and resolution in the classical log-polar representation, resulting in a novel space variant representation.

The last paper of the section, "Graph Kernels from the Jensen-Shannon Divergence" by Bai et al., shows how to construct Jensen-Shannon kernels for graph datasets using the von-Neumann entropy and Shannon entropy.

The third section analyzes textures.

The first paper, "A Simplified Gravitational Model for Texture Analysis" by Junior et al., presents an innovative and efficient methodology for extracting texture information by previously converting an image into a simplified dynamical system in gravitational collapse process.

In "Adaptive Matrices and Filters for Color Texture Classification" by Giotis et al., an integrative approach towards color texture classification and recognition is introduced. The approach is created using Generalized Learning Vector Quantization and adaptive filter kernels based on Gabor filters.

The final section of this special issue focuses on applications related to photometric properties of images, humans' walk and 3D faces, and medical imaging.

"Estimating Photometric Properties from Image Collections" by Diaz et al. presents the highly ill-posed problem of jointly estimating the scene illumination, the radiometric camera calibration and the reflectance properties of an object using a set of images from a community photo collection. The main tools used are appropriate representations of illumination, a non-linear relationship between irradiance and intensity and some surface reflectance properties. 
The next paper, "Spatial Recurrences for Pedestrian Classification" by Serra-Toro et al., develops a framework for pedestrian classification based on spatial recurrences in the form of recurrence plots.

In "3D Deformable Super-Resolution For Multi-Camera 3D Face Scanning" by Ouji et al., a 3D face acquisition solution consisting of a hybrid stereovision and phase-shifting approach is provided.

Using histograms as discrete representations of local statistics for motion analysis, "Histogram-based Optical Flow for Motion Estimation in Ultrasound Imaging" by Tenbrinck et al. designs a new optical flow method for echocardiographies, which is robust under the presence of speckle multiplicative noise.

The last paper, "A Diffeomorphic Mapping Based Characterization of Temporal Sequences: Application to the
Pelvic Organ Dynamics Assessment" by Rahim et al., develops a contour-based dynamic characterization of temporal sequences of pelvic organs based on the large deformation diffeomorphic mapping paradigm and the shape representation based on currents.

The Guest Editor would like to thank the authors of the submitted papers and the valuable referees from the computer vision/pattern recognition communities. Their important work made this special issue possible. Last but not least, our appreciation goes to the Editor-in-Chief, Joachim Weickert, who believes in this initiative and supports the publication of this special issue.

We hope to focus the attention of the computer vision/pattern recognition communities on the latest advances in these areas. 